\title{
A Novel 3D Nonstationary Channel Model Based on the von Mises-Fisher Scattering Distribution
}

\author{
Yuming Bi, Jianhua Zhang, Ming Zeng, Mengmeng Liu, and Xiaodong Xu \\ Key Laboratory of Universal Wireless Communications, Beijing University of Posts and Telecommunications, \\ Ministry of Education, Mailbox No. 92, Beijing 100876, China
}

Correspondence should be addressed to Yuming Bi; biyuming10507@sina.com

Received 26 December 2015; Revised 29 February 2016; Accepted 24 March 2016

Academic Editor: David W. Matolak

Copyright (C) 2016 Yuming Bi et al. This is an open access article distributed under the Creative Commons Attribution License, which permits unrestricted use, distribution, and reproduction in any medium, provided the original work is properly cited.

In the last decade, the nonstationary properties of channel models have attracted more and more attention for many scenarios, that is, vehicle-to-vehicle (V2V), mobile-to-mobile (M2M), and high-speed train (HST). However, little research has been done on the real-physical channel model. In this paper, we propose a generalized three-dimensional (3D) nonstationary channel model, in which the scatterers are assumed to be distributed around the transmitter (Tx) and receiver ( $\mathrm{Rx})$ on a two-sphere model. By employing the von Mises-Fisher distribution, the mean values of the azimuth angle of departure (AAoD) and elevation angle of departure (EAoD) and the azimuth angle of arrival (AAoA) and elevation angle of arrival (EAoA) are tracked by time-variant (TV) Brownian Markov (BM) motion paths, which ensure the nonstationarity of the proposed channel model. Moreover, the TV autocorrelation function (ACF) and Doppler power spectrum density (DPSD) of the proposed nonstationary channel model are calculated by using signal processing tools, for example, fast Fourier transform (FFT) and short-time Fourier transform (STFT). In addition, the simulation results show that the TV scatterer distribution results in a nonstationary nonisotropic channel model, and the proposed model can be employed to simulate the 3D nonstationary channel model.

\section{Introduction}

The transmission channel is one of the most crucial parts in mobile communication systems, while the generation of an accurate and effective channel model for testing of communication systems represents a particular challenge. The majority of existing channel models in the literature rely on the assumption of wide-sense stationary (WSS) and uncorrelated scattering (US) $[1,2]$. Under such an assumption, different transmission delays stay uncorrelated and the second-order channel moments are stationary. However, the physical channel in the real world is nonstationary [2-4]. Furthermore, plenty of empirical and analytical reports reveal that the WSSUS assumption is valid only for a short travelling distance. Therefore, in order to develop a more accurate and suitable channel model for the real-physical channel, it is essential to study nonstationarity channel modeling, which is a newly explored research field.

The channel modeling can be classified into three categories, that is, deterministic, geometry, and correlation statistics
[4]. In terms of deterministic channel modeling, $\mathrm{Ai}$ and $\mathrm{He}$ have applied their measurement data to V2V and HST scenarios employing the nonstationary channel modeling. In [2, 3], the stationary region especially has been extracted according to the correlated matrix distance. This is beneficial to elicit the extent of the channel variation if one could reconstruct the nonstationary channel simulators to approximate the realphysical channel. For geometry methods, [5] proposed a twodimensional (2D) evolutionary spectrum approach to model nonstationary broadband mobile fading channels. In $[6,7]$, the nonstationary properties of massive MIMO channels have been characterized by cluster evolution (birth-death process) on both the array and time axes. Besides, in [8,9], Pätzold suggested a nonstationary one-ring scattering channel model by utilizing Brownian random movement paths of the mobile station and deriving the angle of arrival (AoAs) and angle of departure (AoDs). In addition, [10] provided a generic method to find the MIMO correlation channel matrices under nonstationary interferences. However, on the one hand, all methods mentioned above only took into account 


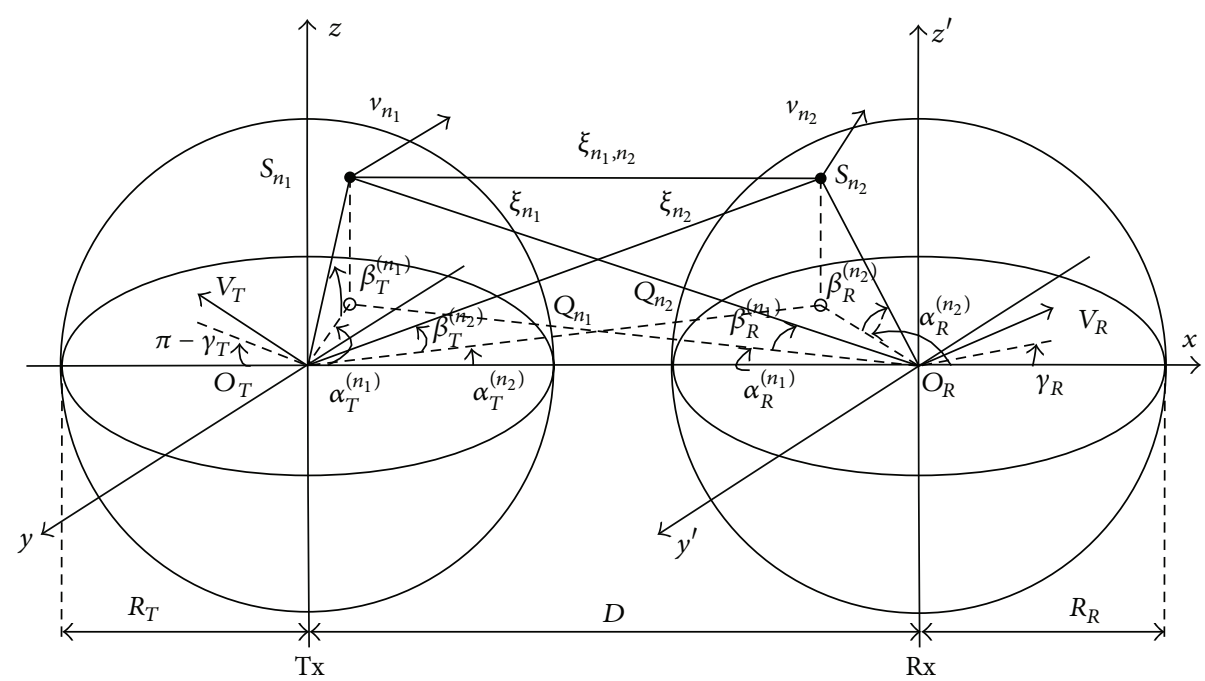

FIGURE 1: The proposed 3D two-sphere channel model.

the nonstationarity of the vertical plane while they ignored the impact of elevation plane; on the other hand, their modeling process required a high computational complexity and lacked flexibility. Therefore, it is essential to develop a simple and feasible 3D channel model for nonstationary research and to extend the $3 \mathrm{D}$ nonstationary channel model to capture the real-physical channel characteristics.

In this paper, we propose a more general $3 \mathrm{D}$ geometric situation where the scatterers are assumed to be distributed around the user in a sphere area. By employing the von MisesFisher scattering distributions, the centers of the AoDs and AoAs scattering distribution are tracked by TV Brownian motion paths [11, 12], which could effectively reflect the movement process of the MS. Furthermore, the ACF of the complex channel gain and local PSD of the Doppler frequencies are calculated by nonstationary signal processing tools, for example, STFT and FFT $[13,14]$. In addition, the results show that the time-variation of the scatterer distribution results in a nonstationary nonisotropic channel model and that the proposed model has the outstanding ability to describe vital statistical characteristics of nonstationary channel, such as the envelope distribution and the TV ACF and PSD [15]. Moreover, it also offers the advantages of low computational complexity and easy realization [8].

The remainder of this paper is organized as follows: Section 2 discusses a novel 3D channel model based on the geometrical two-sphere model. In Section 3, the von MisesFisher (VMF) distribution is employed to describe the $3 \mathrm{D}$ scatter distribution. A time-variant Brownian motion process is provided in Section 4. And then Section 5 proposes a novel $3 \mathrm{D}$ nonstationary channel model. The related simulation and numerical analysis are presented in Section 6. At last, the conclusion is drawn in Section 7.

\section{A Novel 3D Channel Model}

Let us consider a narrowband V2V communication channel with omnidirectional antennas. The radio propagation environment around the transmitter and the receiver is characterized by $3 \mathrm{D}$ nonisotropic scattering under line-of-sight (LoS) and none line-of-sight (NLoS) conditions. Figure 1 illustrates the novel 3D geometrical channel model, in which the local scatterers are modeled on the surface of two spheres of radii $R_{T}$ and $R_{R}$ [16]. In order to limit the computational complexity of this model, both local scatterers around the $\mathrm{Tx}$ and $\mathrm{Rx}$ are considered. Therefore we assume that there are $N_{1}$ scatterers on the sphere (around the Tx) of radius $R_{T}$ and the $n_{1}$ th $\left(n_{1}=1,2, \ldots, N_{1}\right)$ scatterer is denoted by $S_{n_{1}}$. Similarly, around the $\mathrm{Rx}, N_{2}$ scatterers lie on a surface of sphere of radius $R_{R}$, and the $n_{2}$ th scatterer is denoted by $S_{n_{2}}$. The geometric symbols in Figure 1 have the following meaning: $\alpha_{R}^{\left(n_{i}\right)}$ and $\beta_{R}^{\left(n_{i}\right)}$ denote the main AAoA and main EAoA of the $n_{i}$ th scatterer, respectively. In the same way, $\alpha_{T}^{\left(n_{i}\right)}$ and $\beta_{T}^{\left(n_{i}\right)}$ denote the main AAoD and main EAoD of the $n_{i}$ th scatterer, respectively, and $D$ is the distance between the $\mathrm{Tx}$ and Rx. It is worth noting that the distance $D$ is assumed to be much larger than the radius $R_{R}$ or $R_{T}$ (i.e., $R_{T}$ or $R_{R} \ll D$ ). Therefore, the channel impulse response (CIR) of the twosphere model at the carrier frequency $f_{c}$ can be expressed as [17]

$$
h(t)=h^{\mathrm{LOS}}(t)+h^{\mathrm{NLOS}}(t)
$$

where

$$
\begin{aligned}
h^{\mathrm{LOS}}(t)= & \sqrt{\frac{K}{K+1}} e^{-j 2 \pi f_{\mathrm{c}} \tau_{1}} \\
& \times e^{j 2 \pi f_{T \max } t \cos \left(\alpha_{T}^{\mathrm{LOS}}-\gamma_{T}\right) \cdot \cos \beta_{T}^{\mathrm{LOS}}} \\
& \times e^{j 2 \pi f_{R \max } t \cos \left(\alpha_{R}^{\mathrm{LOS}}-\gamma_{R}\right) \cdot \cos \beta_{R}^{\mathrm{LOS}}} .
\end{aligned}
$$


The CIR especially of two-sphere model under NLoS conditions is constructed as a sum of the single- and doublebounced rays with different energies. So it can be rewritten as

$$
\begin{aligned}
h^{\mathrm{NLOS}} & (t)=\sum_{i=1}^{2} h^{\mathrm{SB}_{i}}(t)+h^{\mathrm{DB}}(t) \\
h^{\mathrm{SB}_{i}}(t) & \\
= & \sqrt{\frac{\eta_{\mathrm{SB}}}{K+1}} \lim _{N_{i} \rightarrow \infty} \sum_{n_{i}=1}^{N_{i}} \frac{1}{\sqrt{N_{i}}} e^{j\left(\varphi_{n_{i}}-2 \pi f_{c} \tau_{2}\right)} \\
& \times e^{j 2 \pi f_{T \max } t \cos \left(\alpha_{T}^{\left(n_{i}\right)}-\gamma_{T}\right) \cdot \cos \beta_{T}^{\left(n_{i}\right)}} \\
& \times e^{\mathrm{j} 2 \pi f_{R \max } t \cos \left(\alpha_{R}^{\left(n_{i}\right)}-\gamma_{R}\right) \cdot \cos \beta_{R}^{\left(n_{i}\right)}}, \\
= & \sqrt{\frac{\eta_{\mathrm{DB}}^{\mathrm{DB}}}{K+1}} \lim _{N_{1}, N_{2} \rightarrow \infty_{n_{1}, n_{2}=1}} \sum_{N_{1}, N_{2}} \frac{1}{\sqrt{N_{1} N_{2}}} e^{j\left(\varphi_{n_{1}, n_{2}}-2 \pi f_{c} \tau_{3}\right)} \\
& \times e^{j 2 \pi f_{T \max } t \cos \left(\alpha_{T}^{\left(n_{1}\right)}-\gamma_{T}\right) \cdot \cos \beta_{T}^{(n 1)}} \\
& \times e^{j 2 \pi f_{R \max } t \cos \left(\alpha_{R}^{\left(n_{2}\right)}-\gamma_{R}\right) \cdot \cos \beta_{R}^{\left(n_{2}\right)}}
\end{aligned}
$$

with

$$
\begin{aligned}
\alpha_{T}^{\mathrm{LOS}} & \approx \beta_{T}^{\mathrm{LOS}} \approx \beta_{R}^{\mathrm{LOS}} \approx 0, \\
\alpha_{R}^{\mathrm{LOS}} & \approx \pi, \\
\tau_{1} & \approx \frac{D}{c}, \\
\tau_{2} & \approx \frac{\left(\xi_{n_{i}}+R_{T / R}\right)}{c}, \\
\tau_{3} & \approx \frac{\left(\xi_{n_{1}, n_{2}}+R_{T}+R_{R}\right)}{c},
\end{aligned}
$$

where $\mathrm{SB}_{1}$ and $\mathrm{SB}_{2}$ stand for the subcomponents of singlebounced rays from the Tx sphere and Rx sphere, respectively. $\mathrm{DB}$ means the double-bounced rays. $\eta_{\mathrm{SB}_{i}}$ and $\eta_{\mathrm{DB}}$ are the factors of normalized power, which specify the amount powers of single- and double-bounced rays contribute to the total normalized power. Herein, $K$ is the Rician factor and $c$ denotes the speed of light, and $\varphi_{n_{i}}$ and $\varphi_{n_{1}, n_{2}}$ are independent and identically distributed (i.i.d.) random variables with uniform distribution over $[-\pi, \pi)$ [17]. In addition, $f_{T \max }$ and $f_{R \max }$ represent the maximum Doppler frequencies of the transmitter and the receiver, respectively. $\gamma_{T}$ and $\gamma_{R}$ stand for moving direction of the $\mathrm{Tx}$ and $\mathrm{Rx}$, respectively. The statistical properties and performances of the proposed $3 \mathrm{D}$ $\mathrm{V} 2 \mathrm{~V}$ channel model will be analyzed as follows.

2.1. Envelope and Phase. Multiple uncorrelated fading processes bring the complex envelope, which can be written as

$$
h(t)=h_{i}(t)+j h_{q}(t) .
$$

Considering the distribution of the channel envelope $\xi(t)=$ $|h(t)|$ and phase $\phi(t)=\arg \{h(t)\}$, their corresponding probability density functions (PDF) can be written as [6]

$$
\begin{aligned}
p_{\xi}(z)= & \frac{z}{\delta_{0}^{2}} e^{-\left(z^{2}+k_{0}^{2}\right) / 2 \delta_{0}^{2}} \cdot I_{0}\left(\frac{z k_{0}}{\delta_{0}^{2}}\right), \\
p_{\phi}(\phi)= & \frac{e^{-k_{0}^{2} / 2 \delta_{0}^{2}}}{2 \pi} \\
& \cdot\left\{1+\frac{k_{0}}{\delta_{0}} \cdot \sqrt{\frac{\pi}{2}} \cos \left(\phi-\arg \left\{h^{\mathrm{LOS}}(t)\right\}\right)\right\},
\end{aligned}
$$

where $z$ represents the amplitude variable, the symbol $k_{0}$ is defined as $k_{0}=\sqrt{K /(K+1)}$, and $I_{0}(\cdot)$ is the zeroth-order modified Bessel function of the first kind, while $\delta_{0}^{2}$ stands for the mean power of the channel.

2.2. ACF and PSD. The normalized ACF between any two complex fading envelopes is defined as

$$
r_{h h}(\tau)=\frac{E\left[h(t) \cdot h^{*}(t-\tau)\right]}{\sqrt{E[|h(t)|]^{2} \cdot E[|h(t-\tau)|]^{2}}},
$$

where $(\cdot)^{*}$ is the complex conjugate operation and $E[\cdot]$ represents the statistical expectation operator. Then, we obtain the ACF of the proposed channel model. Taking the LoS and NLoS components into account, the equation of the ACF can be written as

$$
r_{h h}=r_{h h}^{\mathrm{LOS}}(\tau)+\sum_{i=1}^{2} r_{\mathrm{SB}_{i}}^{\mathrm{NLOS}}(\tau)+r_{\mathrm{DB}}^{\mathrm{NLOS}}(\tau)
$$

where

$$
\begin{aligned}
& r_{h h}^{\mathrm{LOS}}(\tau)=\frac{K}{K+1} e^{j(2 \pi / \lambda) \cdot 2 D} \\
& \cdot e^{j 2 \pi \tau\left(f_{T \max } \cos \gamma_{T}-f_{R \max } \cos \gamma_{R}\right)}, \\
& r_{\mathrm{SB} i}^{\mathrm{NLOS}}(\tau)=\frac{\eta_{\mathrm{SB}_{i}}}{K+1} \iint_{-\pi}^{\pi}\left[e^{j 2 \pi \tau\left(f_{T \max } U^{(i)}+f_{R \max } V^{(i)}\right)}\right] \\
& \cdot f\left(\alpha_{T / R}^{\left(n_{i}\right)}, \beta_{T / R}^{\left(n_{i}\right)}\right) d\left(\alpha_{T / R}^{\left(n_{i}\right)}, \beta_{T / R}^{\left(n_{i}\right)}\right), \\
& r_{\mathrm{DB}}^{\mathrm{NLOS}}(\tau)=\frac{\eta_{\mathrm{DB}}}{K+1} \iiint \int_{-\pi}^{\pi}\left[e^{j 2 \pi \tau\left(f_{T \max } U^{\mathrm{DB}}+f_{R \max } V^{\mathrm{DB}}\right)}\right] \\
& \cdot f\left(\alpha_{T}^{\left(n_{1}\right)}, \beta_{T}^{\left(n_{1}\right)}\right) \\
& \cdot f\left(\alpha_{R}^{\left(n_{2}\right)}, \beta_{R}^{\left(n_{2}\right)}\right) d\left(\alpha_{T}^{\left(n_{1}\right)}, \beta_{T}^{\left(n_{1}\right)}\right) d\left(\alpha_{R}^{\left(n_{2}\right)}, \beta_{R}^{\left(n_{2}\right)}\right),
\end{aligned}
$$

where $i$ means there are two subcomponents for singlebounced rays. For example, $i=1$ means the single-bounced rays from the Tx sphere. Similarly, $i=2$ means the single-bounced rays from the Rx sphere. And $f\left(\alpha_{T / R}^{\left(n_{i}\right)}, \beta_{T / R}^{\left(n_{i}\right)}\right)$ stands for the joint azimuth and elevation angle distribution function of the $n_{i}$ th scatterers on $\mathrm{Tx}$ or $\mathrm{Rx}$. According to 
the mathematical law of triangle functions, the geometric relationships between each parameter are defined as

$$
\begin{aligned}
U^{(1)} & =\cos \left(\alpha_{T}^{\left(n_{1}\right)}-\gamma_{T}\right) \cdot \cos \left(\beta_{T}^{\left(n_{1}\right)}\right), \\
V^{(1)} & =\cos \left(\alpha_{R}^{\left(n_{1}\right)}-\gamma_{R}\right) \cdot \cos \left(\beta_{R}^{\left(n_{1}\right)}\right), \\
\alpha_{R}^{\left(n_{1}\right)} & \approx \pi-\frac{R_{T} \cdot \cos \beta_{T}^{\left(n_{1}\right)}}{D} \cdot \sin \left(\alpha_{T}^{\left(n_{1}\right)}\right), \\
\beta_{R}^{\left(n_{1}\right)} & =\arccos \left(\frac{D-R_{T} \cos \beta_{T}^{\left(n_{1}\right)} \cdot \cos \alpha_{T}^{\left(n_{1}\right)}}{\xi_{n_{1}}}\right), \\
\xi_{n_{1}} & =\sqrt{Q_{n_{1}}^{2}+R_{T}^{2} \sin ^{2} \beta_{T}^{\left(n_{1}\right)}}, \\
Q_{n_{1}} & =D+R \cdot \cos \left(\beta_{R}^{\left(n_{1}\right)}\right) \cos \left(\alpha_{R}^{\left(n_{1}\right)}\right), \\
U^{(2)} & =\cos \left(\alpha_{T}^{\left(n_{2}\right)}\right) \cdot \cos \left(\beta_{T}^{\left(n_{2}\right)}\right), \\
V^{(2)} & =\cos \left(\alpha_{R}^{\left(n_{2}\right)}\right) \cdot \cos \left(\beta_{R}^{\left(n_{2}\right)}\right), \\
\alpha_{T}^{\left(n_{2}\right)} & \approx \frac{R_{R} \cdot \cos \beta_{R}^{\left(n_{2}\right)}}{D} \cdot \sin \left(\alpha_{R}^{\left(n_{2}\right)}\right), \\
\beta_{T}^{\left(n_{2}\right)} & =\arccos \left(\frac{D+R_{R} \cos \beta_{R}^{\left(n_{2}\right)} \cdot \cos \alpha_{R}^{\left(n_{2}\right)}}{\xi_{n_{2}}}\right), \\
\xi_{n_{2}} & =\sqrt{Q_{n_{2}}^{2}+R_{R}^{2} \sin ^{2} \beta_{R}^{\left(n_{2}\right)},} \\
Q_{n_{2}} & \approx D+R_{R} \cos \beta_{R}^{\left(n_{2}\right)} \cos \alpha_{R}^{\left(n_{2}\right)}, \\
U^{\mathrm{DB}} & =\cos \left(\alpha_{T}^{\left(n_{1}\right)}\right) \cdot \cos \left(\beta_{T}^{\left(n_{1}\right)}\right), \\
V^{\mathrm{DB}} & =\cos \left(\alpha_{R}^{\left(n_{2}\right)}\right) \cdot \cos \left(\beta_{R}^{\left(n_{2}\right)}\right) .
\end{aligned}
$$

The DPSD has been used for nonisotropic scattering channels and the theoretical expression DPSD is

$$
S_{h h}(f)=\int_{-\infty}^{\infty} r_{h h}(\tau) e^{-j 2 \pi f \tau} d \tau .
$$

\section{3. von Mises-Fisher Distribution}

The VMF distribution $[18,19]$ is a close model for directional data distributed uniformly with rotational symmetry on the unit hypersphere $S^{p-1}$. When $p$ is 3 , the ordinary sphere usually corresponds to the set of all points embedded in the Euclidean space $R^{3}$. The vector $\Omega$ stands for any direction of scatterers on the unit sphere surface and it can be described in Cartesian coordinates as

$$
\Omega=[\cos \beta \cos \alpha, \cos \beta \sin \alpha, \sin \beta]^{T},
$$

where $[\cdot]^{T}$ represents the transpose operation. It is obvious that $\beta$ and $\alpha$ denote the coelevation and azimuth angles of vector $\Omega$, respectively. Then, the general form of the VMF distribution can be written as

$$
f_{p}(\Omega ; \mu, k)=\frac{(k / 2)^{d}}{\Gamma(d+1) \cdot I_{d}(k)} \exp \left(k \mu^{T} \Omega\right),
$$

where $k$ controls the concentration of the distribution about the mean direction vector. In particular, when $k=0$, the distribution is isotropic, and when $k \rightarrow \infty$, the scattering becomes a point source on the surface. Furthermore, parameter $d=p / 2-1$. From a physical point of view, the direction spread represents the degree of direction from the arrival subpaths of a cluster to the mean center of the AoA. Therefore, the mean center value of the AoA indicates the mean direction vector from the MS to the cluster and a subpath shows a multiple within a cluster of scatterers. For the ordinary sphere, the VMF PDF to characterize the distribution of effective scatterers can be defined as

$$
\begin{aligned}
& f(\alpha, \beta) \\
& =\frac{k}{4 \pi \sinh (k)} \\
& \quad \cdot \exp \left\{k\left[\cos \beta_{0} \cos \beta \cos \left(\alpha-\alpha_{0}\right)+\sin \beta_{0} \sin \beta\right]\right\},
\end{aligned}
$$

where $\alpha_{0}$ and $\beta_{0}$ represent the mean values of the azimuth and elevation angle, respectively, $-\pi \leq \alpha_{0} \leq \pi, 0 \leq \beta_{0} \leq \pi$.

\section{TV Brownian Random Process}

In this section, we will bring the temporal Brownian random process [17] to simulate a nonstationary TV $3 \mathrm{D}$ channel model. A standard $\mathrm{BM}$ process $\{B(t): t \in[0, T]\}$ is a Wiener process, in which the increments satisfy a normal and independent distribution. Besides, it needs to satisfy the following conditions:

(1) $B(0)=0$.

(2) When $0 \leq i \leq j \leq T$, the increment $B(j)-B(i)$ obeys the normal distribution with zero mean and variance $j-i$; that is, $B(j)-B(i) \sim N(0, j-i)$

(3) When $0 \leq m \leq n \leq i \leq j \leq T$, the increments $B(m)-B(n)$ and $B(j)-B(i)$ are statistically independent.

Due to the fact that the same statistical properties impact the receiver between the AoA distribution motion and the MS random movement, this paper proposes that the variation of the AoA distribution can be used for simulating a 3D nonstationary channel. To model the motion process of the AoA distribution in the $3 \mathrm{D}$ plane, we provide a path model with BM movement components along the elevation and azimuth plane. The fluctuations of the motion path especially are modeled by two independent temporal BM processes: $B_{t}^{(\alpha)}$ and $B_{t}^{(\beta)}$. Therefore, the motion path can be modeled as

$$
P\left(\alpha_{t}, \beta_{t}\right)=\left\{\begin{array}{l}
\alpha_{t}(t)=\alpha_{s}+w t \cdot \Delta t+\Delta t^{2} \cdot B_{t}^{(\alpha)} \\
\beta_{t}(t)=\beta_{s}+w t \cdot \Delta t+\Delta t^{2} \cdot B_{t}^{(\beta)},
\end{array}\right.
$$

where $w$ stands for the random drift parameter used to control the behavior of the deterministic drift degrees along each axis and $\Delta t$ represents the degree of randomness of the path. Meanwhile, $\Delta t=1 / w ; \alpha_{s}$ and $\beta_{s}$ are the start points of the process along each axis. According to the proof of the 
BM paths model in [17], it is not difficult to find that the mean value of each angle always depends on time $t$ and the ACF is also determined by a function of the time difference $\tau$. Consequently, we can conclude that the BM path model is a nonstationary process. More details about Brownian random process can be found in [17].

\section{The Nonstationary Properties of the Proposed Model}

In this section, we consider the TV CIRs, ACF, and DPSD of the nonstationary process. At first, we introduce the BM process to the VMF scattering distribution, and the TV-VMF PDF can be rewritten as

$$
\begin{aligned}
& f\left(\alpha_{T / R}^{\left(n_{i}\right)}(t), \beta_{T / R}^{\left(n_{i}\right)}(t)\right)=\frac{k}{4 \pi \sinh (k)} \cdot \exp \{k \\
& \cdot\left[\cos \beta_{T / R 0}^{\left(n_{i}\right)}(t) \cdot \cos \beta_{T / R}^{\left(n_{i}\right)} \cos \left(\alpha_{T / R}^{\left(n_{i}\right)}-\alpha_{T / R 0}^{\left(n_{i}\right)}(t)\right)\right. \\
& \left.\left.\quad+\sin \beta_{T / R 0}^{\left(n_{i}\right)}(t) \cdot \sin \beta_{T / R}^{\left(n_{i}\right)}\right]\right\} .
\end{aligned}
$$

Then the TV CIRs of the proposed model in (1)-(3) are obtained when the AoA and AoD become TV variables due to the random motion of the scatterers. Furthermore, the envelope PDF of the modified simulation model is the same as that in (6) and the phase PDF is also presented by (7) because both of them are unrelated with TV frequencies. We consider the TV ACF and DPSD of the nonstationary process [20] as

$$
r_{\widetilde{h} \tilde{h}}(t, \tau)=r_{\widetilde{h} \tilde{h}}^{\mathrm{LOS}}(t, \tau)+\sum_{i=1}^{2} r_{\mathrm{SB}_{i}}^{\mathrm{NLOS}}(t, \tau)+r_{\mathrm{DB}}^{\mathrm{NLOS}}(t, \tau) \text {, }
$$

where

$$
\begin{aligned}
& r_{\widetilde{h} \tilde{h}}^{\mathrm{LOS}}(t, \tau)=\frac{K}{K+1} e^{j(2 \pi / \lambda) \cdot 2 D} \\
& \cdot e^{j 2 \pi \tau\left(f_{T \max } \cos \gamma_{T}-f_{R \max } \cos \gamma_{R}\right)}, \\
& r_{\mathrm{SB}}^{\mathrm{NLOS}_{i}}(t, \tau)=\frac{\eta_{\mathrm{SB}}}{K+1} \iint_{-\pi}^{\pi}\left[e^{j 2 \pi \tau\left(f_{T \max } U^{(i)}+f_{R \max } V^{(i)}\right)}\right] \\
& \cdot f\left(\alpha_{T / R}^{\left(n_{i}\right)}(t),\right. \\
& \left.\beta_{T / R}^{\left(n_{i}\right)}(t)\right) d\left(\alpha_{T / R}^{\left(n_{i}\right)}(t), \beta_{T / R}^{\left(n_{i}\right)}(t)\right), \\
& r_{\mathrm{DB}}^{\mathrm{NLOS}}(t, \tau)=\frac{\eta_{\mathrm{DB}}}{K+1} \iiint \int_{-\pi}^{\pi}\left[e^{j 2 \pi \tau\left(f_{T \max } U^{\mathrm{DB}}+f_{R \max } V^{\mathrm{DB}}\right)}\right] \\
& \cdot f\left(\alpha_{T}^{\left(n_{1}\right)}(t), \beta_{T}^{\left(n_{1}\right)}(t)\right) f\left(\alpha_{R}^{\left(n_{2}\right)}(t),\right. \\
& \left.\beta_{R}^{\left(n_{2}\right)}(t)\right) d\left(\alpha_{T}^{\left(n_{1}\right)}(t), \beta_{T}^{\left(n_{1}\right)}(t)\right) d\left(\alpha_{R}^{\left(n_{2}\right)}(t), \beta_{R}^{\left(n_{2}\right)}(t)\right) .
\end{aligned}
$$

In particular, the parameters in (24)-(25) are given in Section 2. Note that the AoAs $\left(\alpha_{R}(t), \beta_{R}(t)\right)$ and AoDs $\left(\alpha_{T}(t), \beta_{T}(t)\right)$ are applied together with the TV variables [18].

It is noteworthy that the definition of the TV ACF keeps the symmetrical delay characteristic $\tau, r_{\tilde{h} \tilde{h}}(t, \tau)=r_{\tilde{h} \widetilde{h}}(t-\tau)$, and $r_{\tilde{h} \tilde{h}}(t, \tau)$ is the real function if $\widetilde{h}(t)$ is a real process, which

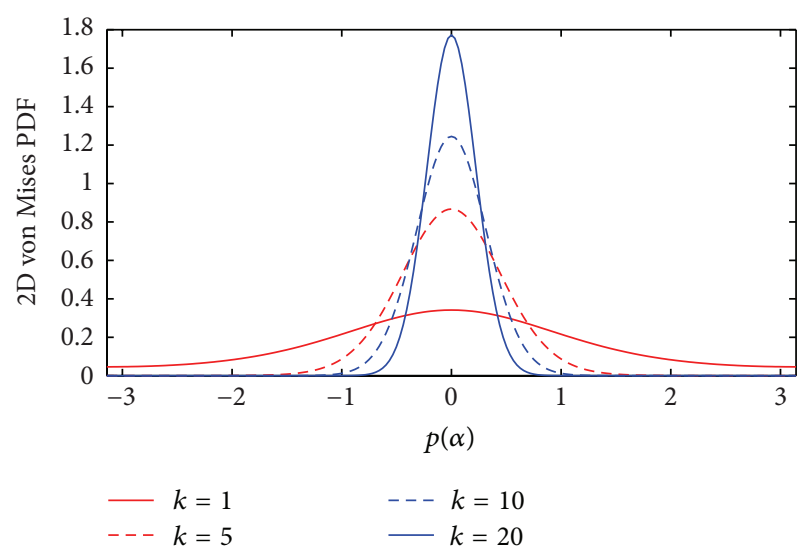

FIGURE 2: 2D VM PDF.

results in the Fourier transform having the symmetrical and real characteristics. Beyond the TV DPSD, the short-time DPSD is widely used to analyze the nonstationary process [17], which is the amplitude's square of the signal's STFT $[21,22]$ :

$$
\operatorname{ST}_{\tilde{h} \tilde{h}}(f, t)=\int_{-\infty}^{\infty} r_{\widetilde{h} \tilde{h}}(t, \tau) e^{-j 2 \pi f \tau} \cdot w(t-\tau) d \tau,
$$

where $(t-\tau)$ is the analytic window slipping with time. The nonstationary process can be viewed as stationary within the analysis window.

\section{Simulation Results and Numerical Analysis}

In this section, simulations are carried out to illustrate the nonstationary properties of our proposed 3D TV channel model based on the VMF scatterer distribution. The impact of the model parameters on the VMF scatterer distribution is investigated first. Then, the nonstationary properties of the proposed two-sphere channel model are evaluated and analyzed in terms of the AoAs and AoDs motion path at the Tx and Rx, the TV ACF of the complex channel gain, and the TV DPSD.

Firstly, we consider the performance of the VMF scatterers distribution. From Figure 2, it is not difficult to find that the parameter $k$ controls the shape of the distribution. Meanwhile, Figure 4 shows the VMF PDF in $3 \mathrm{D}$ coordinates by setting the mean angles $\alpha_{0}=90^{\circ}$ and $\beta_{0}=45^{\circ}$. It is clear that the two-dimensional (2D) von Mises (VM) PDF is derived from the 3D VMF PDF for azimuth angle $\alpha$ with $\beta=0^{\circ}$. Furthermore, the scatterer distribution based on the VMF PDF has been shown in Figure 3. Assuming scatterer distribution is dependent on the axis of symmetry specified by the $z$-axis, it can be seen that the larger the value of $k$, the higher the density near the $z$-axis. Especially when $k=0$, the distribution is isotropic.

Secondly, Figure 5 illustrates a realization of the temporal $\mathrm{BM}$ motion path where $k=1, w=10$, and $\Delta t=0.01$. As seen from this figure, the trajectories of $\alpha_{0}^{(i)}(t)$ and $\beta_{0}^{(i)}(t)$ are varying with time, respectively. The properties of 

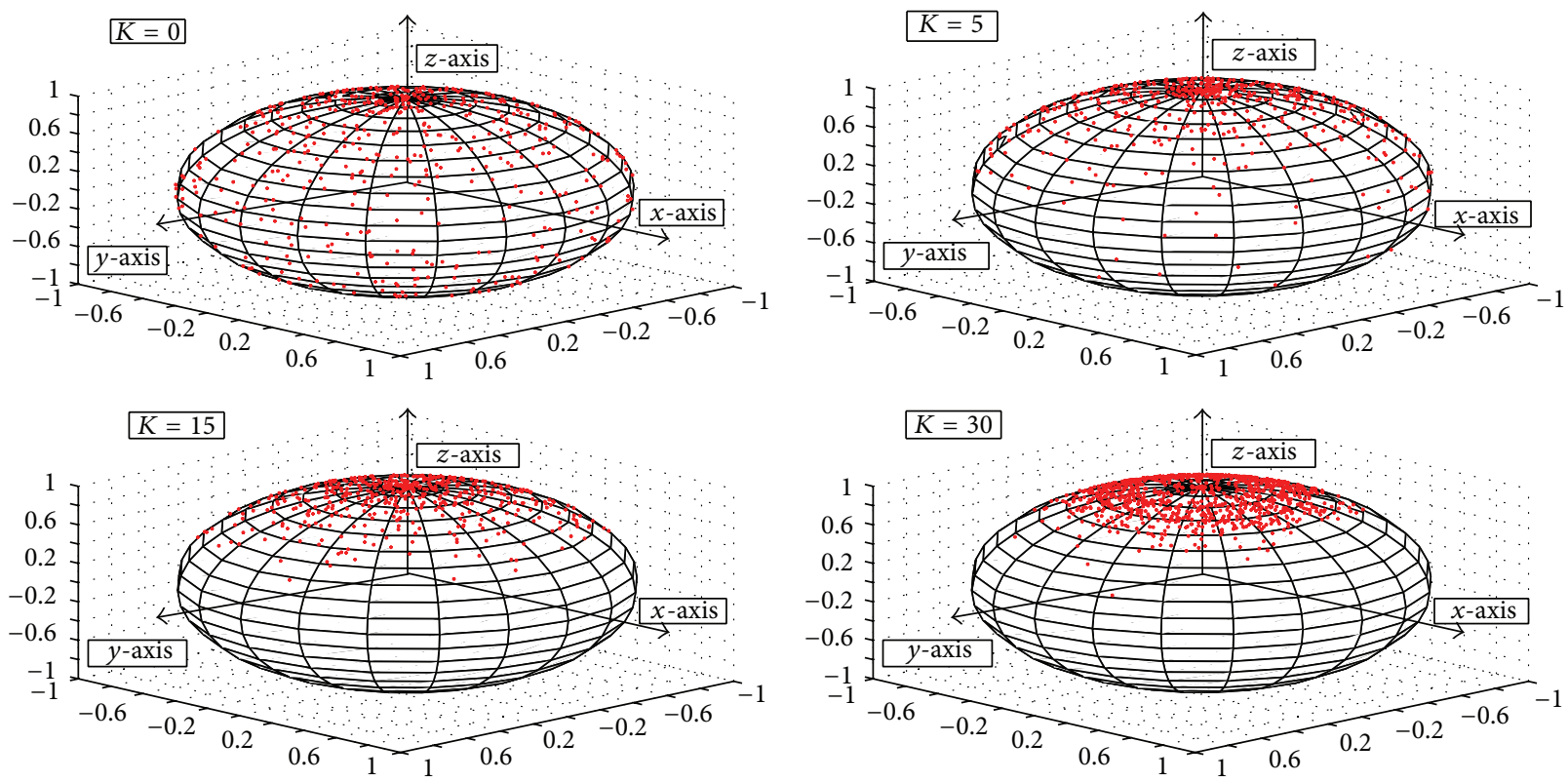

FiguRE 3: Effect of the concentration parameter.

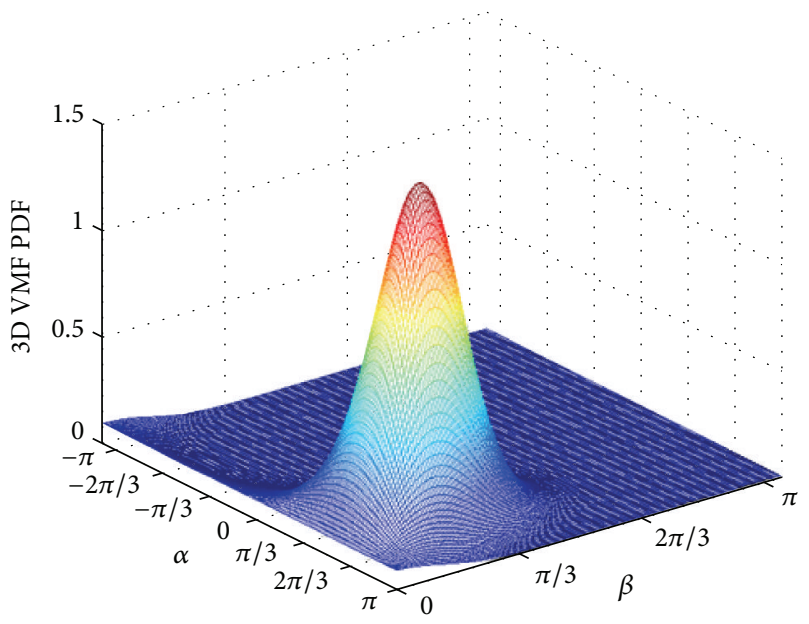

Figure 4: 3D VMF PDF.

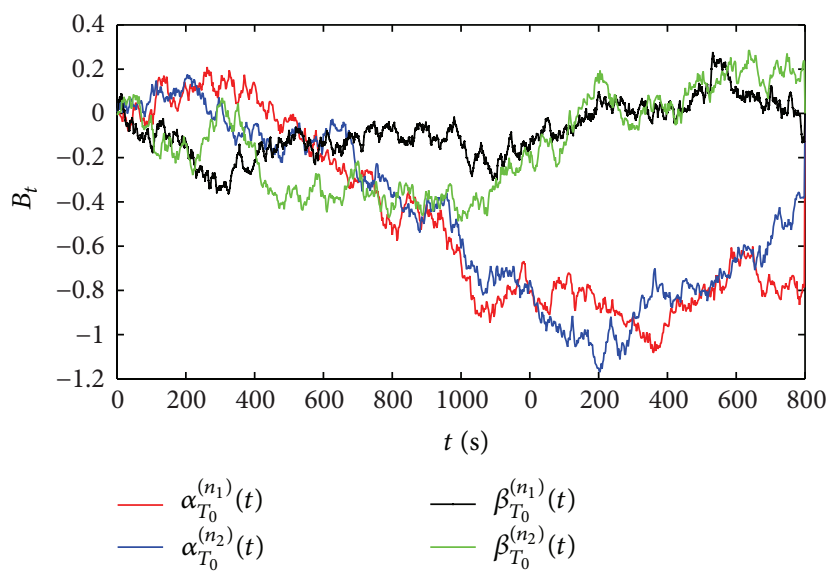

Figure 5: The simulation of Brownian paths. the $\mathrm{BM}$ process prove that the proposed path results in a nonstationary nonisotropic channel model.

Thirdly, Figures 6-8 show the proposed TV 3D models in detail for each statistical value. The simulation settings are as follows: $f_{c}=5.9 \mathrm{GHz}, D=300 \mathrm{~m}, f_{T \max }=f_{R \max }=570 \mathrm{~Hz}$, $R=25 \mathrm{~m}, \gamma_{T}=\gamma_{R}=0^{\circ}, \alpha_{R 0}^{\left(n_{1}\right)}=147.8^{\circ}, \beta_{R 0}^{\left(n_{1}\right)}=17.2^{\circ}$, $\alpha_{T 0}^{\left(n_{2}\right)}=31.6^{\circ}$, and $\beta_{T 0}^{\left(n_{2}\right)}=171.6^{\circ}$. Considering the vehicular traffic density (VTD) based on [4], we have $K=3.876, k=$ 3.6, $\eta_{\mathrm{SB}_{1}}=0.625, \eta_{\mathrm{SB}_{2}}=0.225$, and $\eta_{\mathrm{DB}}=0.15$ for low and $K=0.156, k=0.6, \eta_{\mathrm{SB}_{1}}=0.15, \eta_{\mathrm{SB}_{2}}=0.225$, and $\eta_{\mathrm{DB}}=0.625$ for high VTD scenarios because both parameters $K$ and $k$ are related to the distribution of scatterers (normally, larger values of $k$ corresponding to less dense moving vehicles.).

Figure 6 validates the absolute values of the temporal ACF for the proposed 3D channel model in both low and high VTD scenarios. It can be observed that the temporal ACF is affected by the VTD; namely, the temporal ACF in low VTD scenarios is always higher than that in high VTD scenario. Additionally, Figures 7 and 8 depict the envelope and the phase PDF of 3D channel model determined by the parameters $K$ and $k$, respectively.

Finally, we assume that the mean elevation and azimuth angles are moving along the TV-BM motion paths shown in Figure 5 and we set the maximum Doppler frequency of the transmitter and receiver as $570 \mathrm{~Hz}$. Meanwhile, the effect of the proposed TV-BM motion path on the scattering model is the same as the one in which the vehicle moves randomly in different directions on the AoAs and AoDs. Therefore, as shown in Figures 9 and 12, the absolute value of the resulting local ACF ((24) and (25)) is illustrated. According to these figures, it is manifest that the shapes of the ACF change with different values of time which is due to the nonstationarity of the TV AoAs and AoDs motion processes. It has been proven that the AoAs and AoDs motion processes based on 


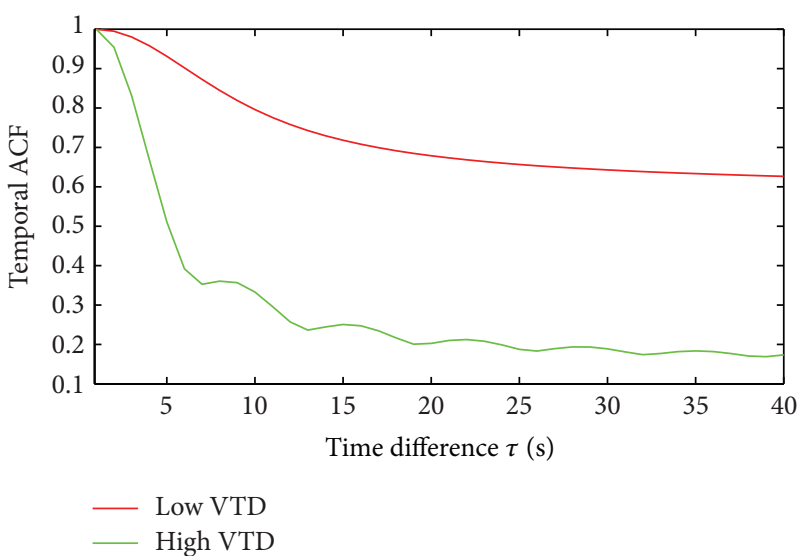

FIgURE 6: The absolute values of the temporal ACF for the proposed 3D TV channel model.

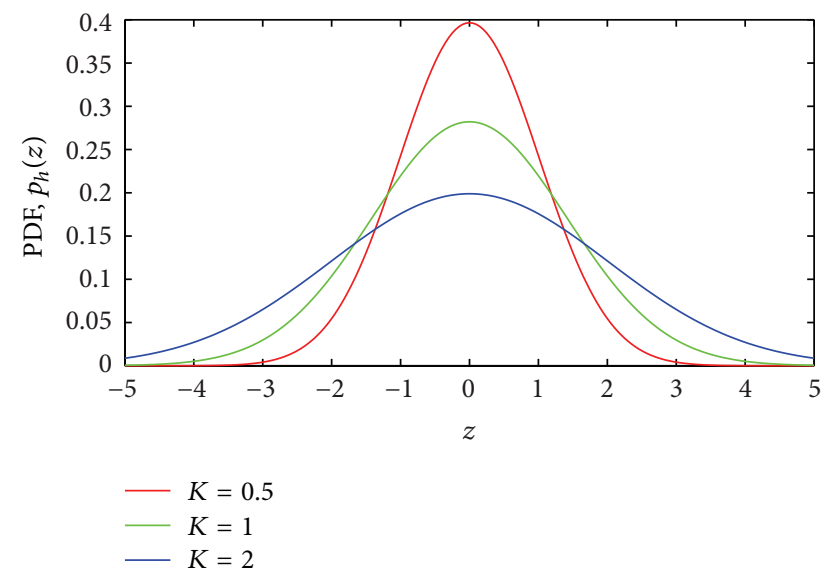

Figure 7: The PDF of amplitude.

the TV-BM path model are first-order nonstationary in [17]. For the same reason, the TV PSD, which is derived from the local ACF by utilizing the FFT with respect to time delay $\tau$, is illustrated in Figures 10 and 13 for low and high VTD scenarios, respectively. Both of them show that the similar Ushape of the PSD varies with time $t$. And this is another way to display the nonstationary properties of the proposed channel model. In addition, the short-time (ST) DPSD $\operatorname{ST}_{\widetilde{h} \tilde{h}}(f, t)$ (see (26)) is shown in Figures 11 and 14. Compared with the FFT DPSD and ST DPSD, we can conclude that the nonstationary properties of them are very similar to each other. This means the STFT has the ability to process the nonstationary channel model. In addition, by comparing the nonstationary properties of the channel model in the low and high VTD scenarios, it can be found that the DPSD derived from the low VTD scenarios is closer to the ideal Jakes DPSD (U-shape spectrum). That is because the effect of nonstationary properties of channel model is more obvious in the high VTD assumption scenarios.

\section{Conclusion}

In this paper, a novel 3D nonstationary channel model for $\mathrm{V} 2 \mathrm{~V}$ is proposed. By employing the proposed temporal

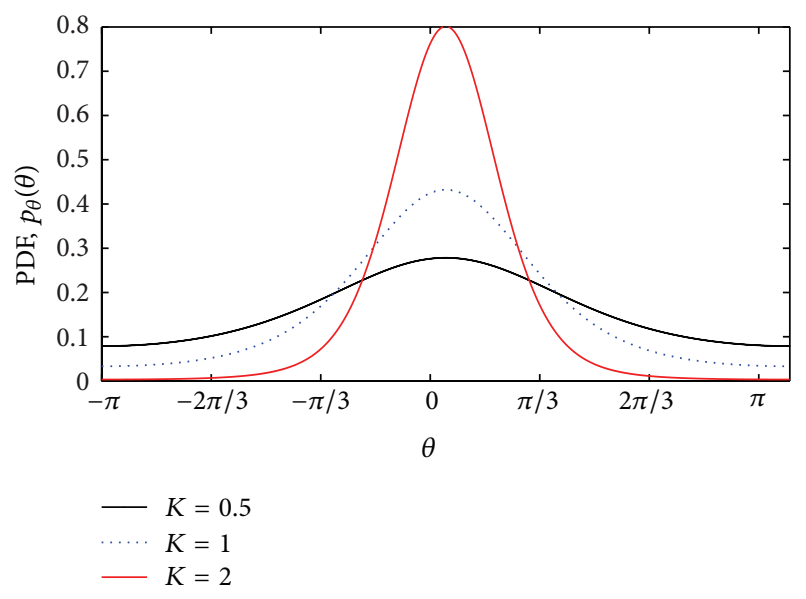

Figure 8: The PDF of phase.

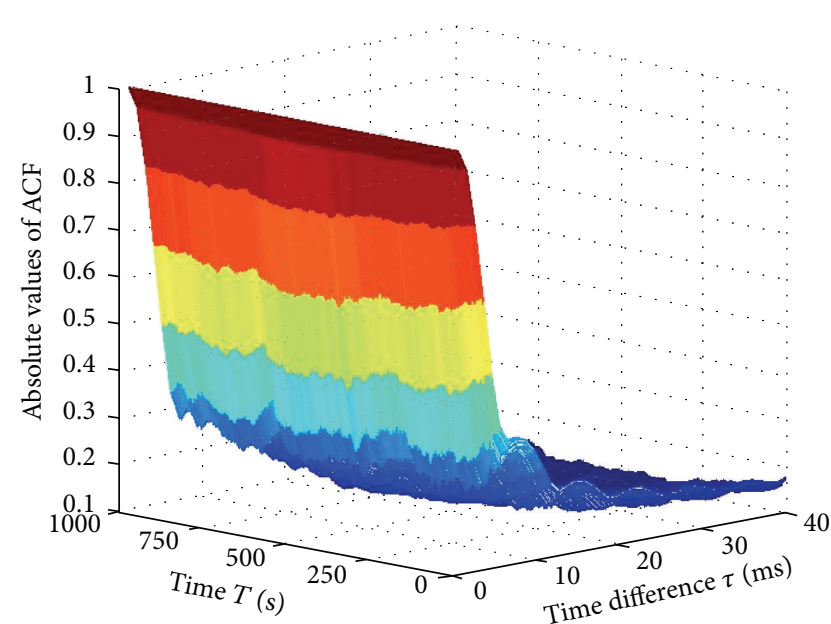

Figure 9: Time-variant ACF.

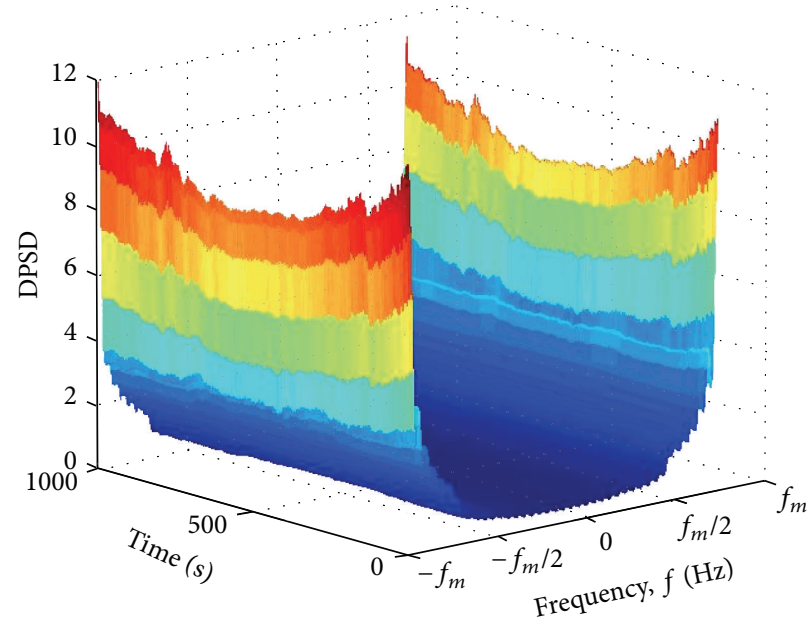

FIGURE 10: Time-variant DPSD (FFT for high VTD). 


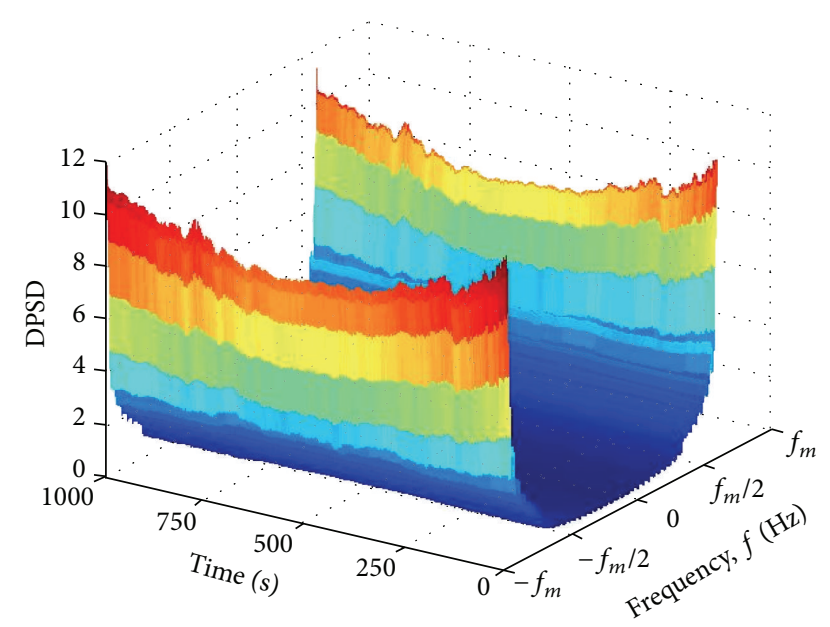

FIGURE 11: Time-variant DPSD (STFT for high VTD).

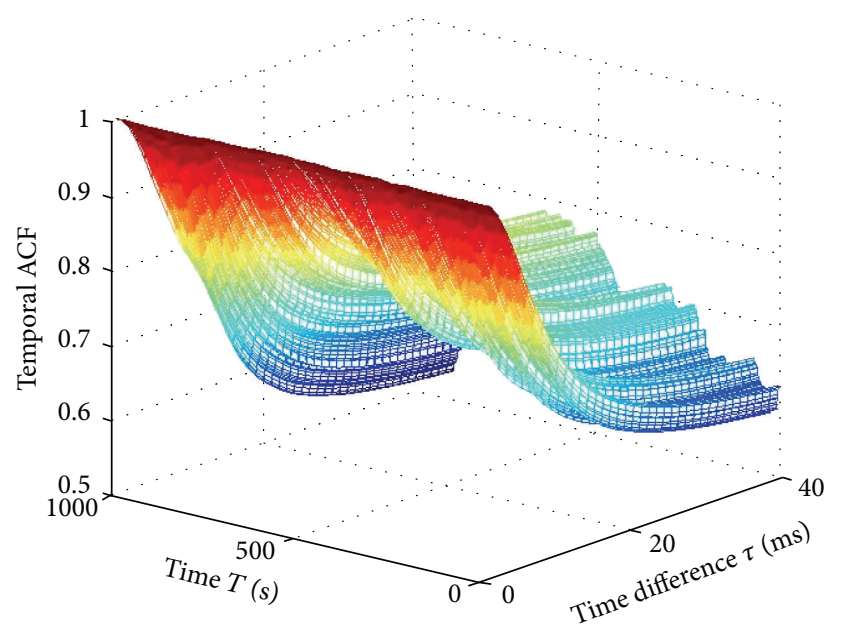

FIgURE 12: Time-variant ACF (low VTD).

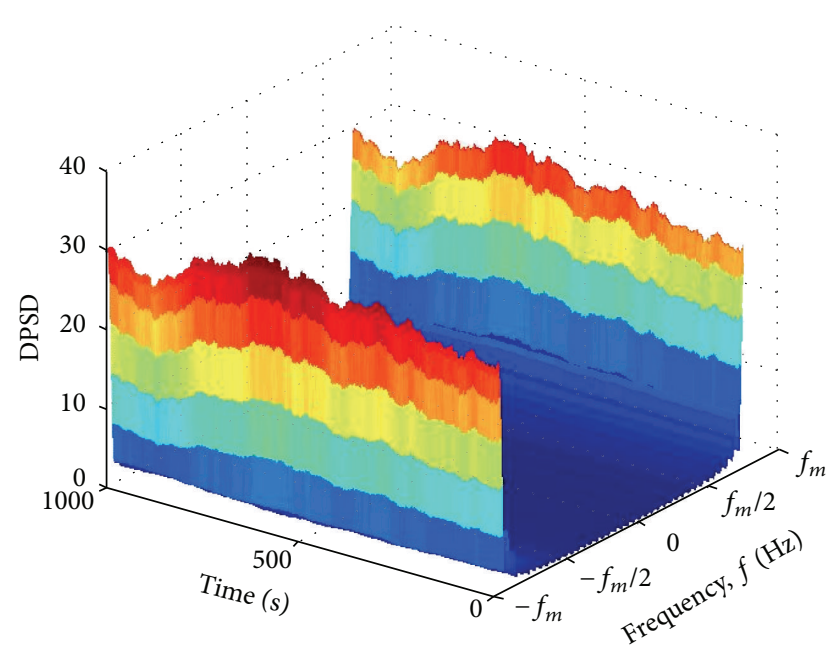

Figure 13: Time-variant DPSD (FFT for low VTD).

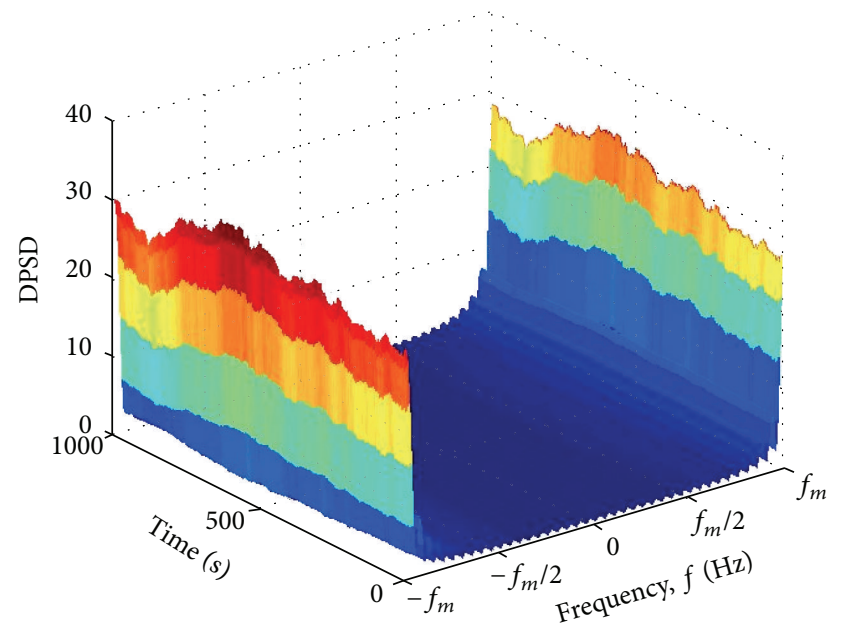

FIgURE 14: Time-variant DPSD (STFT for low VTD).

BM process with the VMF PDF scatterer distribution, we derive a nonstationary nonisotropic channel model which can be applied to simulate the $3 \mathrm{D}$ channels in real time. Moreover, the dynamic changes of the local ACF and PSD have been provided. Lastly, the nonstationary properties of the proposed channel model are verified by simulation. In particular, we have proven that the STFT is also valid for analyzing nonstationary channel models.

\section{Competing Interests}

The authors declare that they have no competing interests.

\section{Acknowledgments}

This research is supported in part by China Important National Science and Technology Specific Projects (no. 2013ZX03001020-002), by the National Key Technology Research and Development Program of China (no. 2012BAF14B01), by the National Natural Science Foundation of China (no. 61171105 and no. 61322110), by the 863 Program Project (no. 2015AA01A703), and by the Doctor Funding Program (no. 201300051100013).

\section{References}

[1] O. Renaudin, V.-M. Kolmonen, P. Vainikainen, and C. Oestges, "Non-stationary narrowband MIMO inter-vehicle channel characterization in the 5-GHz band," IEEE Transactions on Vehicular Technology, vol. 59, no. 4, pp. 2007-2015, 2010.

[2] O. Renaudin, V.-M. Kolmonen, P. Vainikainen, and C. Oestges, "Wideband measurement-based modeling of inter-vehicle channels in the 5-GHz band," IEEE Transactions on Vehicular Technology, vol. 62, no. 8, pp. 3531-3540, 2013.

[3] R. He, Z. Zhong, B. Ai, J. Ding, Y. Yang, and A. F. Molisch, "Short-term fading behavior in high-speed railway cutting scenario: measurements, analysis, and statistical models," IEEE Transactions on Antennas and Propagation, vol. 61, no. 4, pp. 2209-2222, 2013. 
[4] A. F. Molisch, F. Tufvesson, J. Karedal, and C. F. Mecklenbräuker, "A survey on vehicle-to-vehicle propagation channels," IEEE Wireless Communications, vol. 16, no. 6, pp. 12-22, 2009.

[5] Z. Chen, Q. Wang, D. Wu, and P. Fan, "Two-dimensional evolutionary spectrum approach to nonstationary fading channel modeling," IEEE Transactions on Vehicular Technology, vol. 65, no. 3, pp. 1083-1097, 2016.

[6] Y. Yuan, C.-X. Wang, X. Cheng, B. Ai, and D. I. Laurenson, "Novel 3D geometry-based stochastic models for non-isotropic MIMO vehicle-to-vehicle channels," IEEE Transactions on Wireless Communications, vol. 13, no. 1, pp. 298-309, 2014.

[7] S. Wu, C.-X. Wang, E.-H. M. Aggoune, M. M. Alwakeel, and Y. He, "A non-stationary 3-D wideband twin-cluster model for 5 G massive MIMO channels," IEEE Journal on Selected Areas in Communications, vol. 32, no. 6, pp. 1207-1218, 2014.

[8] A. Borhani and M. Pätzold, "Correlation and spectral properties of vehicle-to-vehicle channels in the presence of moving scatterers," IEEE Transactions on Vehicular Technology, vol. 62, no. 9, pp. 4228-4239, 2013.

[9] A. Chelli and M. Pätzold, "A non-stationary MIMO vehicleto-vehicle channel model derived from the geometrical street model," in Proceedings of the IEEE 74th Vehicular Technology Conference (VTC Fall '11), pp. 1-6, IEEE, San Francisco, Calif, USA, September 2011.

[10] K. Guan, Z. Zhong, B. Ai, and T. Kürner, "Deterministic propagation modeling for the realistic high-speed railway environment," in Proceedings of the IEEE 77th Vehicular Technology Conference (VTC '13), pp. 1-5, Dresden, Germany, June 2013.

[11] D. P. Gaillot, E. Tanghe, P. Stefanut et al., "Accuracy of specular path estimates with ESPRIT and RiMAX in the presence of measurement-based diffuse multipath components," in Proceedings of the 5th European Conference on Antennas and Propagation (EUCAP '11), pp. 3619-3622, April 2011.

[12] A. G. Zajič and G. L. Stüber, "Three-dimensional modeling, simulation, and capacity analysis of space-time correlated mobile-to-mobile channels," IEEE Transactions on Vehicular Technology, vol. 57, no. 4, pp. 2042-2054, 2008.

[13] L. Chang, J. Zhang, X. Li, and B. Liu, "Channel estimation and performance analysis for MIMO-OFDM in doubly-selective channels," in Proceedings of the 15th International Symposium on Wireless Personal Multimedia Communications (WPMC '12), pp. 505-509, September 2012.

[14] A. Borhani and M. Paetzold, "Modelling of non-stationary mobile radio channels using two-dimensional Brownian motion processes," in Proceedings of the International Conference on Advanced Technologies for Communications (ATC '13), pp. 241-246, Ho Chi Minh City, Vietnam, October 2013.

[15] A. Borhani and M. Patzold, "A unified disk scattering model and its angle-of-departure and time-of-arrival statistics," IEEE Transactions on Vehicular Technology, vol. 62, no. 2, pp. 473485, 2013.

[16] W. Chen, J. Zhang, Z. Liu, and Y. Bi, "A geometrical-based 3D model for fixed MIMO BS-RS channels," in Proceedings of the IEEE 26th Annual International Symposium on Personal, Indoor, and Mobile Radio Communications (PIMRC '15), pp. 502-506, Hong Kong, August 2015.

[17] A. Borhani and M. Patzold, "A highly flexible trajectory model based on the primitives of brownian fields-part I: fundamental principles and implementation aspects," IEEE Transactions on Wireless Communications, vol. 14, no. 2, pp. 770-780, 2015.

[18] K. Mammasis, P. Santi, and A. Goulianos, "A three-dimensional angular scattering response including path powers," IEEE
Transactions on Wireless Communications, vol. 11, no. 4, pp. 1321-1333, 2012.

[19] K. Mammasis, R. W. Stewart, and J. S. Thompson, "Spatial fading correlation model using mixtures of von mises fisher distributions," IEEE Transactions on Wireless Communications, vol. 8, no. 4, pp. 2046-2055, 2009.

[20] H.-P. Lin, M.-J. Tseng, and F.-S. Tsai, "A non-stationary hidden Markov model for satellite propagation channel modeling," in Proceedings of the IEEE 56th Vehicular Technology Conference (VTC-Fall '02), pp. 2485-2488, Vancouver, Canada, September 2002.

[21] A. G. Zajić and G. L. Stüber, "Three-dimensional modeling and simulation of wideband MIMO mobile-to-mobile channels," IEEE Transactions on Wireless Communications, vol. 8, no. 3, pp. 1260-1275, 2009.

[22] X. Cheng, Q. Yao, C.-X. Wang et al., "An improved parameter computation method for a MIMO V2V rayleigh fading channel simulator under non-isotropic scattering environments," IEEE Communications Letters, vol. 17, no. 2, pp. 265-268, 2013. 

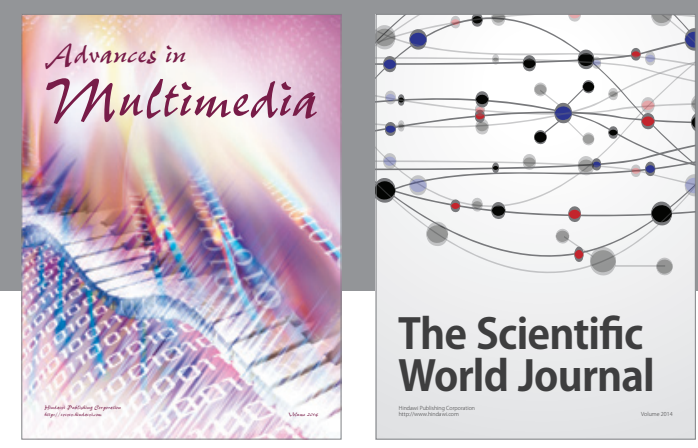

The Scientific World Journal
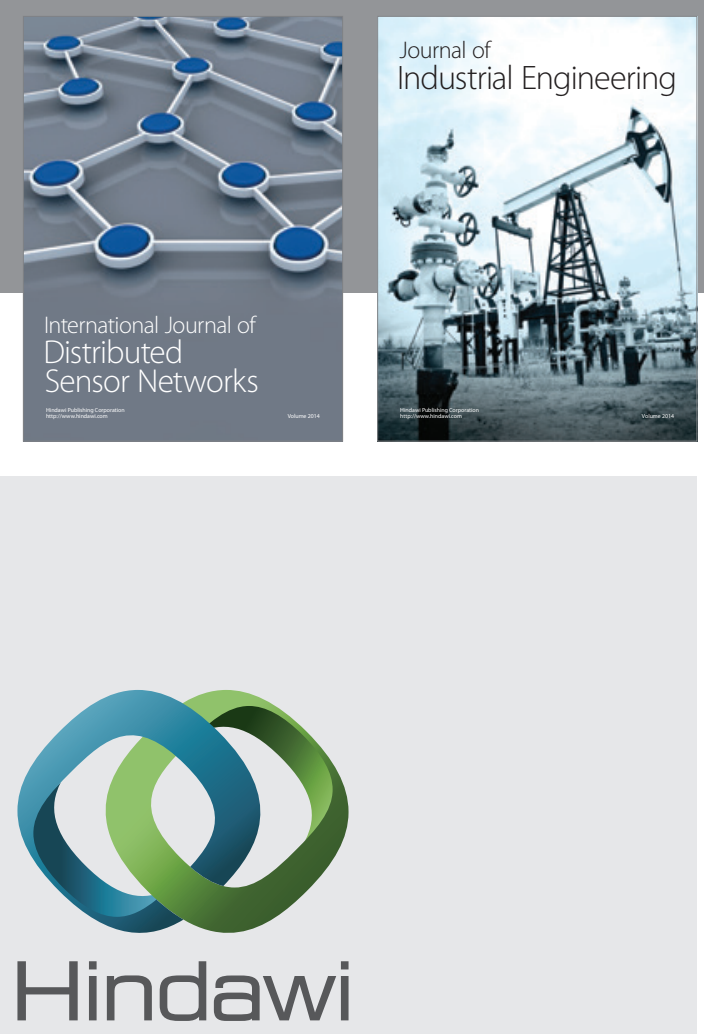

Submit your manuscripts at

http://www.hindawi.com

\section{Computer Networks} and Communications
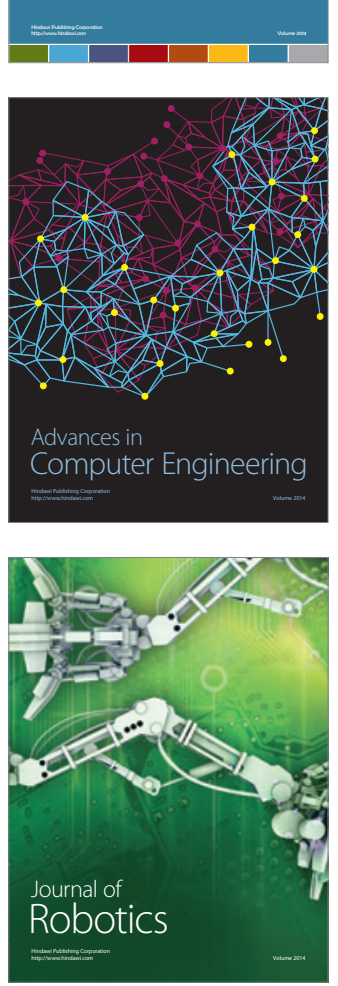
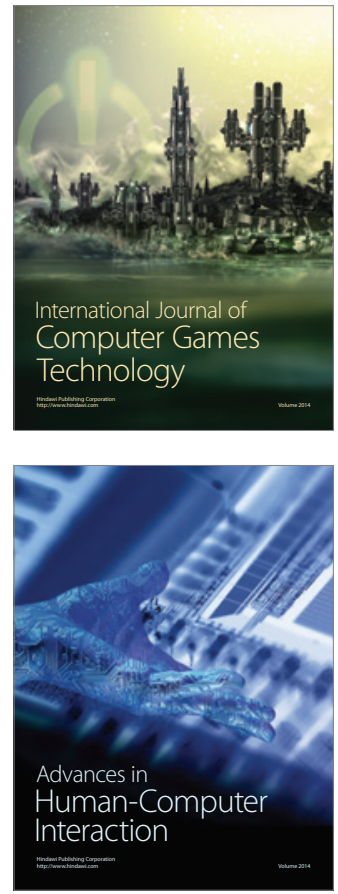
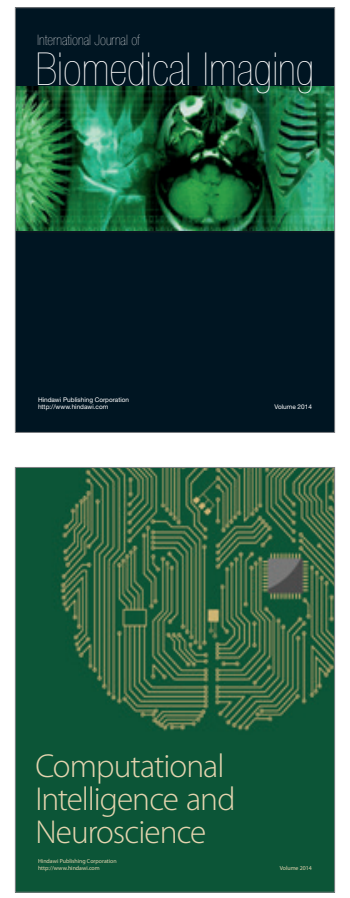
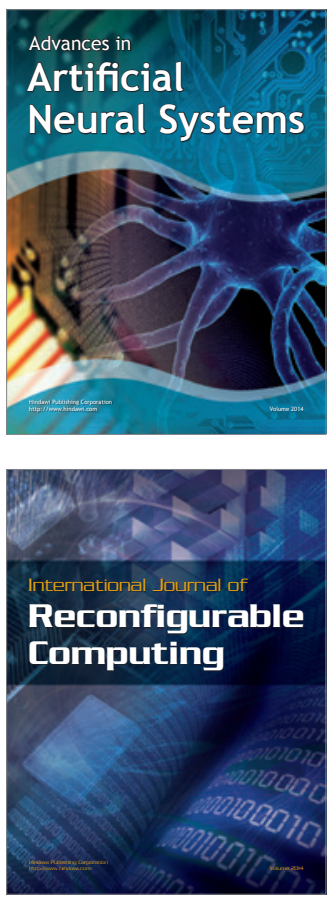
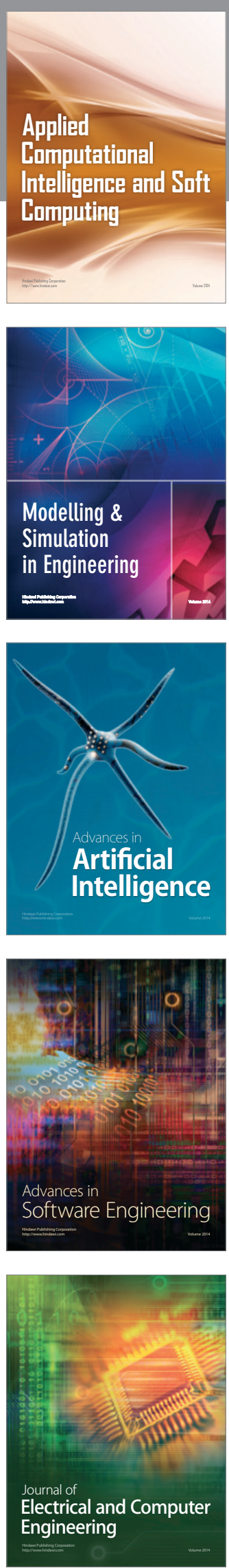